\title{
EL ESTUDIO DE JAPONESISMOS AISLADOS (II): «NIPÓN», «TOQUIOTA», «MISUBISI», «TOIOTA», «SUDOCU», «SUNAMI»
}

\author{
THE STUDY OF ISOLATED JAPANESE LOANWORDS (II) «NIPÓN», \\ «TOQUIOTA», «MISUBISI», «TOIOTA», «SUDOCU», «SUNAMI»
}

\author{
Rafael Fernández Mata \\ Universidad de Córdoba \\ rafaelfernandezmata@gmail.com
}

Fecha de recepción: 11-01-2018

Fecha de aceptación: 07-06-2018

\section{RESUMEN:}

Dentro del inventario de japonesismos utilizados en el español actual, convinimos en reunir dentro de un grupo, a modo de "cajón de sastre", seis unidades que, por pertenecer a áreas referenciales diversas, no podían constituir un amplio conjunto de estudio: «nipón», «toquiota», «misubisi», «toiota», «sudocu», «sunami». En el presente artículo se pretende, por primera vez en lengua española, realizar un estudio exhaustivo de estos seis vocablos ${ }^{1}$, atendiendo a diferentes aspectos lingüísticos (formal, semántico e histórico), además de indicar su grado de adaptación al inventario léxico del español.

Palabras Clave: Japonesismo; nipón; tokiota; mitsubishi; toyota; sudoku; tsunami; lexicografía española; Diccionario de la lengua española.

${ }^{1}$ Dos de estas unidades, «nipón» y «sunami», fueron estudiadas por KNOwLton (1959: 663-664 y 735-736) —ofrece datos históricos_ y PRIETO (2007: 256 y 291-292) —con información acerca de la etimológica y uso actual-. 


\section{Abstract:}

After analyzing the Japanese loanwords used in current Spanish, we agreed to gather together a group of six units which belong to different reference areas and which cannot constitute a broad set: «nipón», «toquiota», «misubisi», «toiota», «sudocu», «sunami». For the first time concerning Spanish, these six Japanese loanwords are described thoroughly from a formal, semantic and historical perspective in the present article. Further, the degree of adaptation of these loanwords within the Spanish lexical inventory will be indicated.

KEY WORDS: Japanese loanword; nipón; tokiota; mitsubishi; toyota; sudoku; tsunami; Spanish Lexicography; Diccionario de la lengua española.

\section{INTRODUCCIÓN}

En nuestra tesis inédita (Los japonesismos de la lengua española: Historia y transcripción, vid. Fernández Mata, 2015) y en nuestro artículo titulado "Los japonesismos del español actual” (vid. Fernández Mata, 2017) establecimos un inventario de 92 voces de origen japonés utilizadas en la lengua española actual².

A continuación, explicaremos sucintamente el proceso que llevamos a cabo para la creación de dicho inventario: en primer lugar, recurrimos a distintos portales cibernéticos ${ }^{3}$ para comprobar si previamente se había compilado un listado de japonesismos. En nuestra búsqueda nos tropezamos con varios trabajos en los que se ofrece un inventario de japonesismos: Knowlton (1959), Kim (1992), Frago Gracia (1997), Gómez Capuz (2004), Reyes Díaz (2004), Prieto (2007), Cid Lucas (2009), Giménez Folqués (2012)4. También encontramos una breve referencia a varios japonesismos introducidos a través de la lengua portuguesa en el capítulo de «Lusismos» de la Enciclopedia Lingüística Hispánica. Cruzamos los resultados anteriores con los inventarios de 9 obras lexicográficas de corte panhispánico ${ }^{5}$ y obtuvimos una lista de 148 voces tomadas en préstamo de la lengua japonesa. Aplicamos una serie de filtros a dicha lista a fin de evitar repeticiones, ítems de origen no japonés y vocablos cuyo uso era nulo, muy restringido o circunstancial, lo que nos llevó al listado de 92 japonesismos.

\footnotetext{
2 Análisis realizado desde un punto de vista panhispánico.

${ }^{3}$ Google (www.google.es), Academia.edu (http://www.academia.edu/), Infoling (www.infoling.org), la Biblioteca Virtual Miguel de Cervantes (http://www.cervantesvirtual.com/) y Dialnet (http://dialnet.unirioja.es/).

${ }^{4}$ A excepción de Knowlton (1959) y Prieto (2007), ninguno de los investigadores lleva a cabo un estudio exhaustivo.

5 Estas, ordenadas según su año de edición, son: el Diccionario de voces de uso actual (1994), el Diccionario del español actual (1999), la vigésima segunda edición del Diccionario de la lengua española (2001), el Gran diccionario de uso del español actual (2001), el Diccionario de uso del español de América y España (2003), el Nuevo diccionario de voces de uso actual (2003), el Diccionario de uso del español (2008), el Diccionario Clave: diccionario de uso del español actual, y la edición en línea de la vigésima tercera edición del Diccionario de la lengua española (2014).
} 
Teniendo en cuenta parámetros semánticos, nuestro inventario podía dividirse en 13 áreas referenciales, las cuales, de mayor a menor, son las siguientes: artes marciales y deporte (18 unidades), cocina y alimentación (16), guerra y gobierno de la nación (10), artes (8), ocio (7), escritura y literatura (6), vivienda y mobiliario (4), vestimenta (4), filosofía y religión (4), botánica (3), economía (3), medicina (3). En último lugar, a modo de "cajón de sastre", quedaba un conjunto de voces compuesto por 6 elementos de diversa índole: área referencial relativa a la geografía y los gentilicios («nipón» y «toquiota»), a los automóviles («misubisi» — también como droga de diseño- y «toiota»), a un pasatiempo matemático («sudocu») y a desastres naturales («sunami») $)^{6}$.

Por tanto, en esta ocasión, estudiaremos este grupo de japonesismos, para cuyo análisis nuestro artículo se dividirá en tres grandes apartados. En el epígrafe de metodología detallamos cuáles han sido los pasos a seguir para reunir el listado de japonesismos anteriores. Contiene, asimismo, un subapartado en el que explicamos nuestra concepción sobre las etapas de los préstamos. En el tercer punto realizamos el análisis lingüístico de las voces seleccionadas. En último lugar, expondremos las conclusiones de nuestra investigación.

\section{METODOLOGÍA}

Con el objeto de recabar el mayor número de datos posible acerca de la expresión, el contenido, la etimología y la historia de las voces japonesas que acabamos de enumerar, utilizamos diversas fuentes.

Por lo que se refiere a la descripción fonético-gráfica, morfosintáctica y semántica de las palabras, tuvimos en cuenta: por un lado, los corpus de referencia del español (el CORDE, el CREA y el CORPES XXI); por otro, obtuvimos más datos en las siguientes obras lexicográficas españolas, todas de corte sincrónico: DVUA, DEA, DLE 2001 —vigésima segunda edición-, GDUEA, DUEAE, NDVUA, DUE, DClave y DLE 2014 —vigésima tercera edición-. Además, intentamos perfeccionar nuestra descripción examinando de cerca otros diccionarios de lenguas europeas: $M W C D$ y $O E D$ (inglés), $D F L$ y $L P R$ (francés), DHLP y DPLP (portugués) y DOVLI y Zingarelli (italiano).

En cuanto al análisis histórico-etimológico de las voces, consideramos: (a) dos obras españolas fundamentales para el estudio diacrónico (el $D C E C H$ y el buscador en línea del NTLLE) y el capítulo de Álvarez DE Miranda, "El léxico español, desde el siglo XVIII hasta hoy" (vid. referencias bibliográficas); (b) la información etimológica que reflejan los diccionarios españoles (de los nueve descritos solo tomaremos el DLE 2001, el GDUEA, el

\footnotetext{
${ }^{6}$ Al igual que en nuestra tesis inédita, vid. Fernández Mata (2015), en todas aquellas voces de origen japonés que siguen nuestro sistema de transcripción al alfabeto latino, emplearemos comillas latinas («»), escribiendo en cursiva aquellas voces que todavía se consideran extranjerismos. En cuanto a nuestros patrones transcriptivos vid. Fernández Mata (2018). A lo largo de este artículo hemos transcrito todas las voces de origen japonés (nombres propios, obras lexicográficas, ciudades, etc.) siguiendo nuestro método de transcripción, con la única excepción de los nombres propios de los autores que hemos consultado para nuestra bibliografía.

${ }^{7}$ Para las abreviaciones usadas en este artículo, cf. el ANEXo que ofrecemos al final de este.
} 
DUEAE, el DUE, el DClave y el DLE 2014, por ser los únicos que contienen datos acerca de la etimología); (c) los descriptores etimológicos de los diccionarios extranjeros; (d) dos obras lexicográficas japonesas (el Daiyirín y el DaiD).

Después de compilar y examinar dichos datos, trataremos de interpretarlos, para lo que hemos dividido la información de cada voz en 7 párrafos, cada uno relativo a un plano descriptivo:

PRIMER PÁRRAFO, donde se exponen las cuestiones relacionadas con la etimología. Tratamos de responder, por orden, los siguientes aspectos: (1) étimo japonés del que procede y pronunciación japonesa de este; (2) acepciones del étimo en japonés.

SEgundo, TERCER y CUARTO PÁRRAFOS, en los que resumimos las cuestiones sobre la expresión, el contenido y el uso de un determinado japonesismo respectivamente. De este modo, en el segundo párrafo, dedicado a la forma gráfica y a la acentuación, respondemos a preguntas como: (1) forma y variantes gráficas con que ha sido adaptada tal palabra (¿patrón gráfico español, extranjerizante o híbrido?); (2) ¿Influjo o procedencia de otra lengua intermediaria? En el tercer parágrafo, donde examinamos el significado y el área referencial, despejamos los siguientes interrogantes: (1) acepciones en español (¿coincide con el japonés u otra lengua intermediaria?); (2) área referencial. En el cuarto y último apartado, donde se analizan las características morfosintácticas, analizamos: (1) género y número (masculino/femenino, singular/plural); (2) patrones combinatorios (uso con artículo, adjetivos, adverbios, etc.).

QUINTO, SEXTO Y SÉPTIMO PÁRRAFOS, en los que describimos los datos históricos de un determinado vocablo. En el quinto párrafo exponemos: (1) ¿cuándo se tiene constancia del primer registro escrito en lengua española? Bien sea textual (proporcionado por los corpus: CORDE, CREA y CORPES XXI), bien lexicográfico (conseguido en las obras lexicográficas de carácter histórico: DCECH y NTLLE; o actual: DVUA, DEA, DLE 2001, GDUEA, DUEAE, NDVUA, DUE, DClave y DLE 2014); (2) ¿se observa algún tipo de variación formal o significativa desde su primer registro? En el sexto párrafo revisamos brevemente los registros escritos de un japonesismo en las otras lenguas extranjeras y comparamos estos datos con los obtenidos en las obras españolas. Utilizamos, pues, tanto las obras lexicográficas españolas como las extranjeras. En el séptimo y último párrafo, concluimos nuestro estudio con el análisis relativo al fenómeno del préstamo léxico, es decir, valoramos en qué momento de adaptación se encuentra un determinado japonesismo — cf. 2.1.—. Determinamos, asimismo, su repercusión y distribución geográfica en el sistema léxico del español.

\subsection{Etapas de los préstamos}

Aunque creemos, al igual que Gómez CAPUz (1998: 214), que resulta muy complejo diseccionar o «acotar en etapas discretas lo que parece ser un verdadero "continuum" entre el extranjerismo crudo y ocasional y el préstamo usual y perfectamente adaptado», nos basamos en su clasificación tripartita para analizar el momento en que se encuentra un de- 
terminado japonesismo: el momento de transferencia, el momento de asimilación y el momento de madurez (Gómez CAPUz, 1998: 219; 2005: 15) .

La primera etapa o MOMENTO DE TRANSFERENCIA de un préstamo léxico se caracteriza por el uso limitado del vocablo en cuestión, que queda relegado al lenguaje o campo técnico con el que esté relacionado. En lo concerniente a su aspecto formal, la voz prestada entra con su grafía y sonido originales (es precisamente este rasgo gráfico el que desechamos para nuestro modelo teórico, dado que la lengua japonesa no utiliza el alfabeto latino). Tras verse desvinculado de los paradigmas formales nativos, se observa inestabilidad y anarquía formal en la voz prestada, que puede sufrir cambios fonéticos y gráficos esporádicos. Por lo que se refiere al componente semántico, la monosemia regula el proceso, ya que el vocablo es adquirido con un solo uso denotativo y libre de cualquier paradigma semántico o asociación emotiva. Debido a la novedad del término, suele ir acompañado por marcas autonímicas como sinónimos, paráfrasis o explicaciones para su comprensión (GómEz CAPUZ, 1998: 220-222; 2005: 15-16, 29).

En cuanto a la segunda etapa o PROCESO DE ASIMILACIÓn, el extranjerismo demuestra su eficacia neológica y comienza un proceso de integración en el nuevo sistema. Este proceso puede prolongarse en el tiempo y supone una evolución de estatus de un determinado préstamo léxico: desde su condición de extranjerismo hasta llegar a ser préstamo, cuando ya ha sido asimilado formalmente, es ampliamente utilizado por muchos hablantes de la lengua receptora, quienes ya apenas lo sienten como elemento exógeno. El proceso de adaptación o naturalización de un determinado extranjerismo supone la asimilación de este a todos los subsistemas o niveles de análisis de la lengua receptora: por lo que se refiere a los niveles fónico-gráfico y morfológico, el préstamo léxico se adecua paulatinamente a los patrones gráficos de la lengua española y modifica ciertas realizaciones extranjerizantes para adaptarlas a la de los sonidos de la lengua de destino — una asimilación fónica_-; asimismo, adquiere los rasgos morfológicos del español (como el género, el número, etc.). Por otro lado, en cuanto al significado, suelen dejar atrás la monosemia para integrarse en las estructuras semánticas o área referencial de la lengua receptora. A veces, cuando se trata de términos técnicos, se produce una simple adición al inventario léxico del español, mientras que otras, cuando el extranjerismo tiene ciertos valores semánticos con palabras nativas, se puede producir la especialización semántica del término (Gómez CAPUZ, 1998: 223-251; 2005: 17-25).

En la tercera etapa o ETAPA DE EXPLOTACIÓN, MADUREZ Y CREATIVIDAD, el préstamo lingüístico se caracteriza por estar completamente asimilado en todos los niveles, por lo que es percibido como un elemento patrimonial de la lengua receptora. De este modo, queda sometido a la acción dinámica, creativa y neológica del lenguaje, es decir, comienza a generar nuevos elementos como si se tratara de un recurso neológico interno, por lo que sigue mecanismos morfosintácticos (formación de derivados, compuestos híbridos, alteración de compuestos nominales y cambio de categoría gramatical) y semánticos (generalización, metáfora, metonimia) propios de la lengua receptora (Gómez CAPUZ, 1998: 251-254; 2005: 25-27).

\footnotetext{
${ }^{8}$ Tendremos en cuenta, asimismo, las directrices propuestas por Álvarez DE Miranda (2004).
} 
Por lo que se refiere al USO GENERAL de una palabra, realizaremos dos pruebas, dependiendo de si la palabra en cuestión se registra o no en el CORPES XXI: ${ }^{9}$ si la voz aparece en el CORPES XXI, pediremos a la plataforma que nos aporte la frecuencia normalizada del término — buscando siempre como primera opción su significante singular - y su dispersión, a fin de comprobar la repercusión geográfica de este japonesismo en el sistema léxico del español. Para establecer una escala, usaremos los parámetros que fueron desarrollados por el $G D U E A,{ }^{10}$ esto es, las marcas de frecuencia se atienen a los siguientes porcentajes: a) frecuencia circunstancial o no significativa (hasta 0,25 por millón); b) frecuencia baja (desde 0,26 por millón hasta 2,99 por millón); c) frecuencia moderada (desde 3 hasta 10,99 por millón); d) frecuencia notable (desde 11 hasta 25,99 por millón); e) frecuencia alta (desde 26 hasta 75 por millón); f) frecuencia muy alta (más de 75 por millón). Con el fin de obtener un número lo más ajustado posible a la realidad, siempre que sea posible, lanzaremos la búsqueda sin marcar la casilla de «grafía original» en el motor de pesquisa del CORPES XXI, para que este considere también las posibles variantes gráficas de una voz. Por otro lado, si la voz no aparece en el CORPES XXI, ya que el CREA no presenta la posibilidad de visualizar la frecuencia, emplearemos el análisis del GDUEA.

Tendremos, por tanto, todas estas precisiones en cuenta para adscribir un determinado japonesismo a un grupo en particular: el grupo de las voces recién transferidas, ${ }^{11}$ el de las voces en proceso de asimilación y el de las voces patrimoniales. Así, al final de cada estudio pormenorizado de los japonesismos se ofrecerá un razonamiento detallado que describa las causas por las que tal japonesismo queda incluido en un determinado grupo.

${ }_{9}$ Cuya versión de junio de 2016 recoge unos 225 millones de formas (cf. <http://www.rae.es/recursos/bancode-datos/corpes-xxi>; 26/05/2017).

${ }^{10}$ Esta obra lexicográfica fue elaborada a partir de los ejemplos de uso proporcionados por un corpus de 20 millones de palabras. El corpus utilizado como base es ampliamente representativo de la lengua española en España e Hispanoamérica (sin olvidar las áreas hispanohablantes de Estados Unidos), en sus variedades escrita y oral, y en géneros y ámbitos variados, tal cual se describe en la obra de Aquilino Sánchez et al. (1995), Cumbre. Corpus lingüístico del español contemporáneo, Madrid, SGEL.

Por lo que se refiere a su frecuencia de uso, leemos en la descripción aportada por el GDUEA: «Para alcanzar un razonable índice de fiabilidad en estas marcas de frecuencia, se han llevado a cabo determinados cálculos estadísticos relativos al total de formas y palabras presentes en el Corpus Cumbre, a los tramos de frecuencia de las más de 250.000 formas diferentes que registra dicho corpus y a la cantidad de formas abarcadas por cada tramo. Cabe señalar que en torno al $50 \%$ de las voces marcadas pertenece al nivel de 'Frecuencia baja' y que el umbral fijado para que una voz acceda al tramo más bajo de frecuencia ha sido de cinco ocurrencias sobre el total del corpus (equivalentes al 0,25 por millón). Por debajo de este umbral, y en relación con el corpus manejado (de 20 millones de palabras), se ha considerado que cualquier frecuencia debía ser calificada de 'circunstancial' o 'no significativa'».

11 Aunque el factor «tiempo de entrada» sea fundamental, a veces no resulta del todo determinante. Así, leemos en Gómez CAPuz (2005: 15): «El propio criterio cronológico puede fallar. A este respecto, podemos citar la incompleta integración de club y sandwich, dos de los anglicismos más antiguos del español (principios del siglo XIX): a pesar de su antigüedad, presentan bastantes variaciones en su pronunciación (/klu/, /klub/), las cuales se agudizan en el ámbito morfológico de la formación del plural: clubs, clus y clubes, sandwiches y sandwichs». De este modo, el tiempo de introducción será considerado, pero no determinará la inclusión de un elemento léxico en uno u otro grupo: únicamente las pruebas lingüísticas y no las extralingüísticas, que serán coadyuvantes, (como «el tiempo» o «el valor» o «uso general» de los usuarios) serán las que establezcan su cualidad. 


\section{JAPONESISMOS}

\subsection{Geografía-gentilicio}

\subsection{1. «nipón»}

El préstamo «nipón» proviene de una de las dos lecturas, [n'ippon] ${ }^{12}$, de los ideogramas 日本, empleados para designar el nombre del propio país, esto es, 'Japón' (Daiyirín).

En el conjunto de los corpus españoles hay variedad formal: en CORDE, en su función como adjetivo o adjetivo sustantivado, aparece registrado como nipón-nipones, niponaniponas en 49 casos, y solo un caso de transcripción extranjerizante nippón. Por otro lado, hallamos 7 casos como sustantivo sinónimo de Japón, con forma Nifón ${ }^{13}$. En CREA y CORPES XXI se extiende el uso del adjetivo, aunque se regulariza en nipón-nipones, nipona-niponas. Las obras lexicográficas españolas dan cuenta de las formas singulares nipón, nipona (DEA, DLE 2001, GDUEA, DUEAE, DUE, DClave y DLE 2014). En las obras lexicográficas extranjeras, los significantes son diferentes de acuerdo con los patrones morfológicos internos de cada lengua: inglés $\rightarrow$ Nipponese $(M W C D$ y $O E D)$; francés $\rightarrow$ nippon, nipponne o nippone (DFL y LPR); portugués $\rightarrow$ nipônico $(D H L P$ y $D P L P)$; italiano $\rightarrow$ nipponico, nipponica (DOVLI y Zingarelli). En lo referente a su acentuación, no hay dudas, las muestras de los corpus y la información fónica de los diccionarios GDUEA y DClave nos informan de que se trata de una voz con patrón oxítono, similar al de la lengua de origen.

Por lo que respecta a su significado, detectamos consenso en los corpus del español (CORDE, CREA y CORPES XXI) y las obras lexicográficas, españolas (DEA, DLE 2001, GDUEA, DUEAE, DUE, DClave y DLE 2014) y extranjeras (MWCD, OED, DFL, LPR, $D H L P, D P L P, D O V L I$ y Zingarelli). En las lenguas occidentales analizadas se toma como base el significante [n'ippon] —que funciona como nombre propio para designar 'Japón' en japonés - para formar el gentilicio. En las obras lexicográficas españolas encontramos desde una (en DEA, GDUEA, DUE y DClave) hasta dos acepciones (en DLE 2001, DUEAE y $D L E$ 2014). Si bien, en los diccionarios de una acepción, el descriptor semántico fusiona las dos definiciones que aparecen en otros diccionarios; de este modo, como sostiene el DLE 2014, puede emplearse como adjetivo o sustantivo para: 'natural del Japón', o como adjetivo para lo 'perteneciente o relativo a este país de Asia'. Asimismo, se suele utilizar como sinónimo de japonés (DEA, GDUEA, DUEAE, DUE y DClave). Los corpus del español analizados dan cuenta de las acepciones recién expuestas.

\footnotetext{
12 En cuanto a la transcripción fonética que llevamos a cabo en este artículo, ensombreceremos los segmentos vocálicos que, según nuestra percepción, se articulan con mayor intensidad. Nos hemos basado en las muestras de hablantes nativos reales contenidas en el diccionario de pronunciación en línea <http://es.forvo.com/>. [n']: sonido consonántico, coronal, palatalizado, nasal y sonoro (AKAMATsU, 1997: 121-124; VANCE, 2008: 87-88; LABRUNE, 2012: 78).

${ }^{13}$ Cuya $f$ se explica por la transcripción extranjerizante errónea del sistema Hepburn, ya que en este sistema / ho/ se transcribe como ho y no comofo.
} 
En cuanto a su integración en el sistema morfológico del español, hemos comprobado que en las obras lexicográficas españolas (DEA, DLE 2001, GDUEA, DUEAE, DUE, DClave y DLE 2014) aparece como adjetivo masculino o femenino, que, en su acepción como ‘natural del Japón' puede usarse como sustantivo. Su función como adyacente nominal está bien extendida en los corpus (periódico nipón, sociedad nipona, militares nipones, islas niponas), y su función de adjetivo sustantivado aparece junto al artículo (el nipón, la nipona, los nipones, las niponas). Su uso plural parece, asimismo, bastante sólido: con forma nipones para el masculino y niponas para el femenino. En otras lenguas romances este japonesismo también ha pasado como adjetivo o adjetivo sustantivado (DFL, LPR, DHLP, DPLP, DOVLI, Zingarelli). No detectamos preferencia por ninguna combinación sintáctica recurrente.

De acuerdo con los datos del CORDE, el primer registro escrito de esta palabra en español, con función de adjetivo (el dulce idioma nipón), data de 1881 — en la obra argentina de Lucio Vicente López, Recuerdos de viaje-, aunque, con dicha función, no sería inventariada hasta la decimocuarta edición del Diccionario de la lengua castellana, de 1914. Con función sustantiva, sinónimo de 'Japón', su registro es anterior, ya que aparece con la adaptación Nipon o Nifon en la obra lexicográfica de Gaspar y Roig, de 1855. Por lo tanto, es bastante probable que la función adjetiva derive de esta primera forma, ya que en japonés el significante sustantivo, [n'ippon] se empleaba para referirse al nombre propio del país, esto es, 'Japón'14. Más allá del proceso Nifon > nipón que hemos descrito, desde su primer registro escrito no se han detectado variación formal y significativa relevante.

En otras lenguas europeas, su datación es de diversa época: así, en inglés, el $M W C D$ indica que su primer testimonio data de 1673, mientras que el OED lo sitúa, como Nippon, en 1727. En francés, el $L P R$ señala que, como adjetivo, aparece a finales del siglo XIX, pero, como sustantivo referido a 'Japón', su datación es mucho anterior, 1765, con significantes le Nippon o le Niphon. El DHLP afirma que, para el portugués, el primer registro es de 1897-1898. Por último, en italiano, el DOVLI y el Zingarelli coinciden en fechar su primer registro en 1908.

Teniendo en cuenta la información de los párrafos precedentes, creemos que la voz «nipón» pertenece al grupo de los japonesismos que se encuentran totalmente asimilados en lengua española. No presenta inestabilidad gráfica, acentual o morfológica y, aunque su referente sea exógeno, puede resultar tan foráneo como inglés, francés, italiano o portugués, por ejemplo; al respecto, no hemos hallado marcas autonímicas para aclarar o explicar su valor semántico. Asimismo, como sostiene el CORPES XXI, su frecuencia es moderada (nipón: 4,22 casos por millón) y se distribuye geográficamente de la siguiente manera:

\footnotetext{
${ }^{14}$ Esta es la teoría sostenida por el $L P R$ para el francés y por el $D H L P$ para el portugués.
} 


\begin{tabular}{|lrr|}
\hline País & Freq & Fnorm. \\
\hline \hline España & 445 & 5,24 \\
\hline México & 92 & 3,40 \\
\hline Chile & 64 & 4,48 \\
\hline Argentina & 49 & 2,25 \\
\hline Colombia & 41 & 2,39 \\
\hline Cuba & 41 & 4,58 \\
\hline Estados Unidos & 41 & 12,87 \\
\hline Venezuela & 39 & 3,29 \\
\hline Bolivia & 37 & 7,72 \\
\hline Ecuador & 36 & 6,21 \\
\hline
\end{tabular}

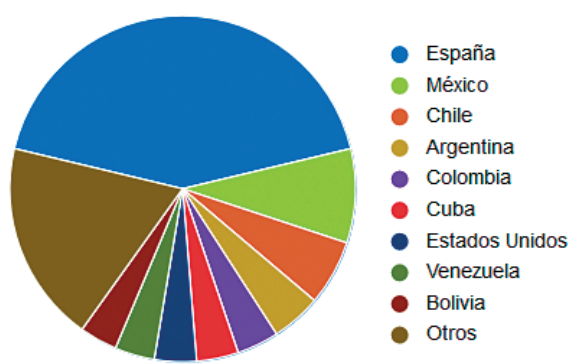

GRÁFICA I.

Distribución por países. Fuente: CORPES XXI.

\subsection{2. «toquiota»}

El gentilicio «toquiota» procede del étimo japonés 東京, [to:k’o: ${ }^{15}$, nombre de 'la capital de Japón', más la suma del sufijo /-ita/ ${ }^{16}$, que, en este caso, pierde la /i/ por disimilación — véase más adelante nuestra teoría-.

En el conjunto de los corpus (CORDE, CREA y CORPES XXI), solo viene recogida la grafía extranjerizante tokiota(s), la preferida por la RAE, según el Diccionario panhispánico de dudas ${ }^{17}$. La única obra lexicográfica que inventaría este significante es el NDVUA. En otras lenguas extranjeras se emplean las grafías: Tokyoite o Tokyo-ite (MWCD y OED), tokyote o tokyoüte (DFL) y tokyota o edochiano (Zingarelli). En cuanto a su acentuación, dado que la voz ha sufrido un proceso de sufijación, no podemos comparar la derivación española con el étimo japonés. No obstante, por las formas aportadas por los corpus, se podría entender que esta voz ha pasado a la lengua española como voz paroxítona.

En cuanto a su significado, detectamos consenso en los corpus del español (CORDE, CREA y CORPES XXI) y las obras lexicográficas, tanto española (NDVUA) como extranjeras (MWCD, OED, DFL y Zingarelli), cuyo significado es el de 'perteneciente a Toquio'.

El NDVUA sostiene que se trata de un adjetivo, sin especificar más sobre sus propiedades morfológicas. Gracias al Diccionario panhispánico de dudas, sabemos que esta forma es "válida para ambos géneros", como observamos en su uso en los corpus: la ex vanguardista tokiota, la bolsa tokiota, el mercado tokiota, del selectivo tokiota, etc. Registramos usos sustantivados, con artículos en singular y en plural: para el tokiota, el civismo de los

15 [k’]: sonido consonántico, dorsal, palatalizado, oclusivo, sordo (AKAMATSU, 1997: 78; VANCE, 2008: 92-93).

16 Según la RAE (2009: 536), el sufijo -ita "es invariable en género" y "propio de los adjetivos gentilicios que aluden a pueblos semitas o cercanos a ellos". En español no presenta connotaciones semánticas despreciativas, como así ocurre en ciertos casos con su correlato inglés (vid. DoI, 2013: 94).

${ }^{17}$ Compruébese en: http://lema.rae.es/dpd/?key=tokiota 
tokiotas, etc. En francés (DFL) e italiano (Zingarelli) también se registran estos dos usos morfológicos. No detectamos preferencia por ninguna combinación sintáctica.

La primera documentación textual de la palabra, en plural, tokiotas, tiene lugar en la década de los 60 del siglo XX, concretamente, según datos del CORDE, en $1964^{18}$ —en el libro español, El Japón y su duende, de José María Gironella-. Desde su primer registro escrito no observamos variación formal o significativa.

Por lo que se refiere a las lenguas extranjeras consultadas, el $O E D$ sitúa su primer registro en 1973 para el inglés. El diccionario Zingarelli ubica su primera aparición en 1991 en italiano. La similitud del significante español (tokiota) con el de las otras lenguas estudiadas (Tokyoite/Tokyo-ite —inglés-, tokyoteltokyoüte — francés- y tokiota -italiano-), nos hizo pensar que tal vez este se debiera al influjo de aquellas. Sin embargo, en español se registra con anterioridad, por lo que pudiera deberse este fenómeno al sustrato de sufijación latino compartido por lenguas tan próximas ${ }^{19}$. En español, pudo suceder lo siguiente: (1) al sustantivo /tókio/ se le añadió el sufijo /-íta/ (-ite en inglés y francés e -ita en italiano), que, según el DLE 2014, procede del latín (-īta o -ītes, y este del griego -ítпs) y se utiliza para formar "principalmente adjetivos gentilicios y otros que expresan pertenencia". (2) Como ocurrió en otros vocablos procedentes del japonés ${ }^{20}$, el sufijo español porta en última instancia la intensidad silábica: /tokioíta/; pero, por disimilación con la vocal /i/ presente en el elemento compositivo principal, /tókio/, la /i/ del sufijo desapareció y cedió su intensidad a la /o/ final de /tókio/. Un fiel reflejo de esta cuestión se puede observar en francés, donde son válidas las dos formas resultantes, una sin disimilación (tokyoüte) y otra con disimilación (tokyote). Parece ser que la disimilación ${ }^{21}$ es el resultado más habitual, pues en italiano también se produjo (tokiota).

Teniendo en cuenta toda la información previa, creemos que «toquiota» pertenece al grupo de los japonesismos totalmente asimilados, a pesar de su grafía extranjerizante y su valor monosémico, ya que se trata de un gentilicio necesario en lengua española para designar a los habitantes de la capital japonesa. Según los datos del CORPES XXI, su repercusión en el sistema léxico del español es circunstancial o no significativa $(0,09$ casos por millón para la forma tokiota) y su distribución en los diferentes países hispánicos es la siguiente:

18 De ese mismo año son los primeros registros que ofrece la Hemeroteca digital de la Biblioteca Nacional de España: http://hemerotecadigital.bne.es/results.vm?o=\&w=tokiota\&f=text\&t $\equiv \% 2$ Bcreation $\& \mathrm{l}=600 \& \mathrm{l}=700 \& \mathrm{~s}=0 \&$ lang $=\mathrm{es}$

${ }^{19}$ En palabras de LeOPARDI, serían "europeísmos", mientras que MALKIEL las denomina "cadenas panrománicas" o "paneuropeas", etiquetas que explica el profesor Álvarez DE MiRANDA (2004: 1041) cuando describe el fenómeno de la "inducción" o "catálisis léxica".

${ }^{20}$ Aunque no hemos incluido su análisis, podemos aducir ejemplos de nuestra tesis como: nipoperuano, fuyimorismo, fuyigolpe, poquemanía, etc.

${ }^{21}$ Además de esta, no podemos olvidar la tendencia histórica del español a deshacer los hiatos — vid. LAPESA (2005 [1981]) o QUILIS (2006 [1993])—. 


\begin{tabular}{|lrr|}
\hline País & Freq & Fnorm. \\
\hline \hline España & 6 & 0,07 \\
\hline Estados Unidos & 4 & 1,25 \\
\hline México & 4 & 0,14 \\
\hline Argentina & 2 & 0,09 \\
\hline Ecuador & 2 & 0,34 \\
\hline Perú & 2 & 0,26 \\
\hline Bolivia & 1 & 0,20 \\
\hline Colombia & 1 & 0,05 \\
\hline Panamá & 1 & 0,48 \\
\hline
\end{tabular}

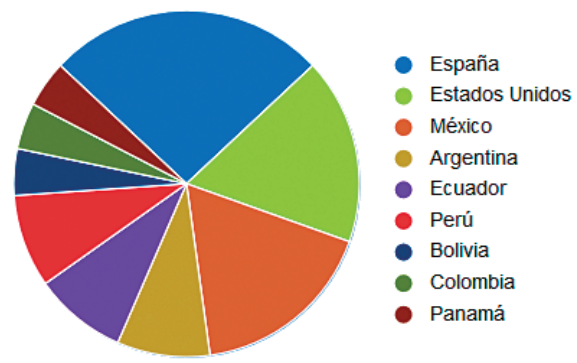

GRÁFICA 2.

Distribución por países. Fuente: CORPES XXI.

\subsection{Automóviles}

\subsection{1. «misubisi»}

La voz «misubisi» procede del étimo 三菱, [m'itsub'is'i] ${ }^{22}$, 'tres diamantes' ${ }^{23}$, nombre de una empresa japonesa cuyo logotipo es: ${ }^{24}$.

Este vocablo se registra en los corpus del español actual (CREA y CORPES XXI) y en una obra lexicográfica española (NDVUA); en todos con la forma extranjerizante mitsubi$s h i$. La fórmula singular con mayúscula inicial, Mitsubishi, de la que se registran más casos, se emplea para referirse al nombre de la compañía automovilística o para el vehículo perteneciente a dicha marca. Por lo que respecta a su acentuación, en lengua española no hay lugar a dudas, tanto el NDVUA como los corpus demuestran que no existen variantes articulatorias de su patrón acentual: esta palabra ha sido adaptada con acentuación llana, por lo que guarda parecido con la lengua de origen.

En lo concerniente al valor semántico de su forma con minúscula inicial, mitsubishi(s), detectamos coincidencia entre los corpus del español (CREA y CORPES XXI) y el NDVUA: este vocablo se utiliza con el significado de 'pastilla de droga'. Por la información del $N D$ $V U A$ sabemos que este japonesismo en lengua española procede de un uso metafórico: esta pastilla de droga de diseño posee "una incisión que reproduce el logotipo de la marca japonesa de automóviles Mitsubishi, tres rombos unidos en el centro por uno de sus ángulos agudos". De este modo, en los corpus encontramos este vocablo junto a otros relaciona-

22 [m']: sonido consonántico, bilabial, palatalizado, nasal, sonoro (AKAMATSU, 1997: 117; VANCE, 2008: 87);

[u] sonido vocálico, posterior, cerrado, sin protrusión labial (TsuJimura, 1996 [2007]: 17; AKAMATSU, 1997 : 31-32; VANCE, 2008: 56-57); [b’] sonido consonántico, labial, palatalizado, oclusivo, sonoro (AKAMATSU, 1997 : 78; VANCE, 2008: 76-77); [s'] sonido coronal, palatalizado, fricativo, sordo (AKAMATsU, 1997: 91-92; VANCE, 2008: 78, 81).

${ }^{23}$ De acuerdo con la información de hablantes nativos.

${ }^{24}$ Obtenido a través del sitio web oficial de la empresa: http://www.mitsubishi.com/e/group/about.html 
dos con el área referencial de las drogas o nombres de drogas de diseño: pastillas, pastilla de ácido, pac man, tiburón, lacoste, versace, éxtasis, etc. No obstante, aunque sin muestras en $C R E A$ - ya que todos los ejemplos aparecen con mayúscula inicial—, en CORPES XXI observamos dos casos colombianos de mitsubishi, en minúscula, para referirse a un vehículo $^{25}$. Si bien, estos casos parecen fortuitos, ya que, como hemos expuesto, con significado de 'vehículo de la marca Mitsubishi' impera la transcripción con mayúscula inicial, tanto en CREA como en CORPES XXI.

En cuanto a su morfología como sustantivo relativo a 'pastilla de droga', resulta inestable - tal vez porque en ocasiones se relaciona con nociones de género femenino (las pastillas) y, en otras, con elementos masculinos (los opiáceos o tripis)—. El NDVUA le asigna género masculino ${ }^{26}$, y así aparece, aunque solo con un ejemplo, en las muestras del CREA: varios mitsubishis. En el mismo autor ${ }^{27}$ que ofrece la forma masculina, detectamos dos casos de sustantivo femenino: las doscientas mitsubishis, las saltarinas mitsubishis. Dado su referente, normalmente agrupado, se utiliza con mayor frecuencia en forma plural, con el morfema de plural /-s/. Por lo que respecta a su morfología plural para 'vehículo de la marca Mitsubishi', prevalece el morfema cero de plural, puesto que solo hallamos un caso puertorriqueño en CREA y otro chileno en CORPES XXI de morfema plural con /-s/. No detectamos preferencia por ningún tipo de patrón combinatorio para ninguna de las nociones semánticas analizadas.

Según los datos del CREA, el primer registro escrito de esta voz, Mitsubishi, hace referencia a la empresa y se fecha el 10 de noviembre de 1976, en el diario español El País (informes procedentes de Tokio señalan que Mitsubishi había abandonado). Por lo que respecta a su valor semántico como 'droga', data de 2002 —en la obra de Boris Izaguirre anotada a pie de página- No obstante, gracias a la muestra del NDVUA, podemos adelantar su primera aparición al periódico madrileño Diario16, el 7 de julio del 2000.

Tras examinar los datos anteriores, creemos que la voz «misubisi» pertenece al grupo de los japonesismos que se encuentran en proceso de transferencia, pues presenta grafía extranjerizante, inestabilidad morfológica, monosemia —en el caso de pastilla, que no de empresa automovilística - y uso en contextos muy restringidos. En este sentido, según el CREA y el CORPES XXI, su repercusión en lengua española es circunstancial o no significativa (0,01 casos por millón). Su uso como epónimo de empresa (Mitsubishi) es panhis-

25 Leemos en CORPES XXI: ese mugroso mitsubishi; la puta alarma del mitsubishi.

${ }^{26}$ No entendemos por qué el NDVUA le asigna género masculino, puesto que en el ejemplo que aporta no aparece junto a adyacentes que indiquen claramente su género. De recurrir a la semántica, está claro que con esta etiqueta se hace referencia a pastilla. Véase el ejemplo del NDVUA: «Con el principio activo del MDMA, que era utilizado por los psiquiatras en los años sesenta para combatir problemas de autoestima, el éxtasis se ha introducido en la juventud para "aguantar la marcha" del fin de semana. Y en muchas discotecas puede detectarse el trapicheo de estas pastillas, de atractivos colores y troqueles ("snoopys", "mitsubishis", "casitas", etcétera), que se llegan a distribuir incluso en la pista de baile al precio de 1.500 a 2.000 pesetas» [realzado nuestro].

27 CREA: Boris Izaguirre (2002): 1965. Madrid: Espasa Calpe. 
pánico $^{28}$ y de frecuencia baja (1,83 casos por millón), mientras que su uso como 'droga' se acota al español europeo (forma mitsubishi) ${ }^{29}$. Nos encontramos, por tanto, ante un japonesismo que ha desarrollado en lengua española, concretamente en su modalidad europea, un nuevo valor semántico.

\subsection{2. «toiota»}

La voz «toiota» procede del étimo トヨタ, [tojota] ${ }^{30}$, denominación de una empresa japonesa cuyo nombre deriva del apellido del fundador, Quichiro Toioda ${ }^{31}$.

Este japonesismo se documenta en los corpus del español actual (CREA y CORPES $X X I)$ y en una obra lexicográfica española (NDVUA), en todos con la forma toyota. Al ser el nombre propio de una empresa, tanto en CREA como en CORPES XXI, observamos un mayor uso de $T$ mayúscula inicial: 491 casos en $C R E A$, frente a 3 de toyota, y 1302 casos en CORPES XXI, frente a 10 de toyota(s). Por lo que respecta a su acentuación, en lengua española no hay lugar a dudas, tanto el NDVUA como los corpus demuestran que no existen variantes articulatorias sobre su patrón acentual: esta palabra ha sido adaptada con acentuación llana, diferente al patrón japonés, que es esdrújulo.

En lo que concierne a su significación, el NDVUA lo acota a simple 'vehículo todoterreno', aunque, como podemos apreciar en la página web de la empresa, son fabricantes de todo tipo de vehículos de cuatro ruedas ${ }^{32}$, como así lo demuestra su uso en los corpus, donde su significación es más amplia, de acuerdo con la fabricación de la firma. También se hace referencia, sobre todo en mayúscula, al nombre propio de la empresa.

Por lo que se refiere a su análisis morfológico, el NDVUA le asigna género masculino, ya que hace referencia, en última instancia, a 'un coche'. Así se recoge en los corpus, donde aparece junto adyacentes masculinos como: el-los, un, etc. Por otro lado, también se registran muestras femeninas, junto a la, una, roja, etc., con las que se denomina bien un 'tipo

28 Gráfica 3. Distribución por países. Fuente: CORPES XXI.

\begin{tabular}{|lrr|}
\hline País & Freq & Fnorm. \\
\hline \hline España & 141 & 1,66 \\
\hline Venezuela & 43 & 3,63 \\
\hline Chile & 41 & 2,87 \\
\hline México & 30 & 1,10 \\
\hline Paraguay & 28 & 5,12 \\
\hline Estados Unidos & 27 & 8,47 \\
\hline Argentina & 26 & 1,19 \\
\hline Ecuador & 23 & 3,96 \\
\hline Bolivia & 17 & 3,54 \\
\hline Colombia & 14 & 0,81 \\
\hline
\end{tabular}

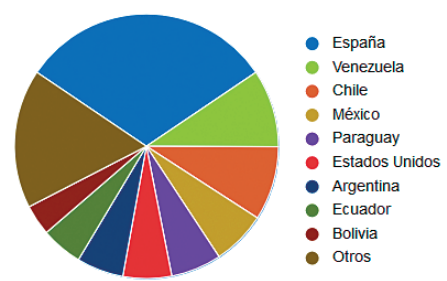

${ }^{29}$ No solo los ejemplos de la obra de Boris Izaguirre (autor venezolano cuya novela se publica en España), sino la muestra que ofrece el CORPES XXI: Guía sobre drogas. Madrid: Delegación del Gobierno para el Plan Nacional sobre Drogas. Ministerio de Sanidad y Consumo, 2007. España

30 [j-] representa un sonido semivocálico, palatal (AKAMATsu, 1997: 43; VANCE, 2008: 89).

${ }^{31} \mathrm{Cf}$. en: http://www.toyota-global.com/company/history_of_toyota/75years/text/taking_on_the_automotive business/chapter1/section1/item1.html

${ }_{32}$ Véase: http://www.toyota.com/espanol/ 
de camioneta', o bien la 'compañía fabricantes de coches'. Cuando su referente es 'coche', el plural es inestable: a veces con morfema plural: los Toyotas —escasos-; otras con morfema cero: los Toyota. Tal vez, en este último caso podríamos suponer la elisión de: los $\underline{\text { co- }}$ ches de la compañía Toyota. Detectamos, asimismo, preferencia por la combinación sintáctica vehículo / carro / automóvil / camioneta + marca Toyota. De esta voz deriva la lexía toyotismo, que significa: 'sistema de organización del trabajo, en el cual el trabajador toma partido en la productividad de la empresa sugiriendo ideas o mejorando la calidad de la producción' (sust. masc. DVUA, NDVUA y DClave).

La compañía fue creada en $1937^{33}$, de modo que este sustantivo tuvo que entrar en español a partir de esta fecha. Si bien, según los datos del CORDE, el primer registro escrito de esta voz data de 1964, con forma Toyota referida a la compañía automovilística ${ }^{34}$ (de nuevo en la obra española de José María Gironella, El Japón y su duende). Su primer uso, como sustantivo equivalente a 'vehículo de dicha marca', está fechada en 1982 y pertenece a la novela española La conspiración del Golfo, de Fernando Schwartz: le adelantaron sucesivamente un Toyota que conducía un beduino. Desde su primer registro escrito no detectamos variación formal — más allá de la escritura con $t$ - minúscula inicial—o significativa.

De acuerdo con la información recién expuesta, creemos que la voz «toiota» pertenece al grupo de los japonesismos que se encuentran a medio camino entre la transferencia y la adopción, pues presenta aún inestabilidad morfología, además, de dos valores semánticos muy restringidos. Contabilizamos los casos con minúscula inicial, para evitar su uso como nombre de marca, y el CORPES XXI indica que su repercusión en el sistema léxico del español es circunstancial o no significativa ( 0,04 casos por millón) y se reparte de la siguiente manera en los diversos países hispánicos:

\begin{tabular}{|lrr|}
\hline País & Freq & Fnorm. \\
\hline \hline El Salvador & 4 & 1,11 \\
\hline Colombia & 2 & 0,11 \\
\hline Panamá & 2 & 0,96 \\
\hline Guinea Ecuatorial & 1 & 1,08 \\
\hline México & 1 & 0,03 \\
\hline
\end{tabular}

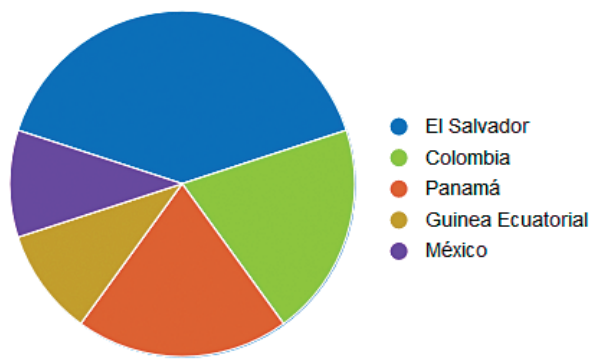

GRÁFICA 4.

Distribución por países. Fuente: CORPES XXI.

33 Véase: http://www.toyota-global.com/company/history_of_toyota/75years/

${ }^{34}$ Leemos en CORDE: «la[s] constructoras de automóviles Toyota». 


\subsection{Pasatiempo matemático}

\subsection{1. «sudocu»}

La voz «sudocu» procede del étimo japonés 数独, [su:doku], utilizado en esta lengua para aludir a 'un tipo de puzle. En un cuadrado de nueve líneas por nueve columnas dividido en bloques de tres líneas por tres columnas, se escriben las cifras del 1 al 9 de modo que no se repitan en ninguna columna, línea o bloque' (DaiD).

Por lo que se refiere a la forma gráfica de la palabra, tanto en CORPES XXI como en DClave y DLE 2014, se transcribe con la grafía extranjerizante sudoku(s), significante empleado también por las obras lexicográficas extranjeras (MWCD, OED, DFL, LPR, $D O V L I$ y Zingarelli). Por la ausencia de tilde en las muestras recogidas en los corpus y la información fónica del DClave, inferimos que el japonesismo ha sido adaptado con patrón acentual paroxítono, diferente al esdrújulo de la lengua de origen, tal vez porque pasó de forma escrita a través del modelo de transcripción inglés, Hepburn, o porque se adecuó a los patrones silábicos de la lengua de destino ${ }^{35}$.

En lo relativo a su valor semántico, los descriptores de los diccionarios españoles (DClave y DLE 2014) y extranjeros (MWCD, OED, DFL, LPR, DOVLI y Zingarelli) coinciden con la significación de la voz en la lengua de origen, esto es, se emplea para denominar el 'pasatiempo de lógica que consiste en completar con números las casillas de una cuadrícula respetando determinadas reglas' (DClave). De este modo, el japonesismo suele aparecer, en CORPES XXI, junto a vocablos relacionados semánticamente con este juego de lógica: descifrar, resolver, crucigramas, ejercicios, solución, dotes lógicas, tablero, ajedrez, cifra, fila, columna, celda, subcuadrícula, etc.

En cuanto a su integración en el sistema morfológico del español, las obras lexicográficas españolas (DClave y DLE 2014) y su empleo en el CORPES XXI lo describen como un sustantivo masculino; por lo que normalmente va acompañado por artículos masculinos (el$l o s, u n)$, conglomerado de preposición y artículo ( $\mathrm{del}$ ) u otros adyacentes (incluido). Como sustantivo masculino ha sido adoptado también en francés ( $F F L$ y $L P R$ ) e italiano (-invariable - DOVLI y Zingarelli). Sus formas plurales, con morfema /-s/, son estables. Aunque no hallamos preferencia por ningún patrón combinatorio, se emplea junto a verbos del tipo: resolver, descifrar y hacer + el / un sudoku.

De acuerdo con la enciclopedia japonesa en línea Chiezo Mini ${ }^{36}$, el auge de este juego de lógica en Occidente se lo debemos a los periódicos británicos, que en 2005 comenzaron a incluirlos en su sección de pasatiempos y a popularizarlos con la etiqueta de sudoku. De este modo, no es de extrañar que, en español, el primer registro escrito tenga lugar, según el CORPES XXI, en 2006, el 9 de julio de 2007 en el periódico argentino en línea Clarín.

\footnotetext{
35 Cf. al respecto LAPESA (2005 [1981]), Quilis (2006 [1993]) — o FradEJAs Rueda (2000).

${ }^{36}$ Compruébese en el portal Kotobank: https://kotobank.jp/word/\%E6\%95\%B0\%E7\%8B\%AC189910\#E3.83.87.E3.82.B8.E3.82.BF.E3.83.AB.E5.A4.A7.E8.BE.9E.E6.B3.89
} 
com (entre los que no faltará el Sudoku). Desde su aparición no observamos cambios en su forma o valor semántico.

En cuanto a las demás lenguas occidentales, en inglés, el $O E D$ fecha el primer registro de la voz en 2000, mientras que el $M W C D$ lo sitúa en 2004. En francés e italiano, su primera aparición es de 2005 (LPR, DOVLI y Zingarelli).

A pesar de su reciente introducción en la lengua española (2006), lo cierto es que la voz presenta un estadio avanzado de asimilación. La grafía extranjerizante y la historia de su introducción en Occidente a través de los periódicos británicos podrían ser causas más que justificadas para creer que la lengua inglesa actuó como intermediaria en su proceso de adopción en lengua española. En cuanto a su significado, es monosémico, aunque bien conocido, pues no presenta ayuda de marcas autonímicas para su explicación, ni tampoco parece quedar vinculado a un referente exógeno a nuestra cultura. Incluso se puede utilizar con valor figurado o metafórico: Los chicos de Mendilibar se quedaron aturdidos, incrédulos, como tantos otros equipos, ante el gran sudoku de la Liga: de nuevo el Madrid por encima sin haber pestañeado; Su mismo nombre, asimilarlo y entender sus aristas, resultaba un difícil sudoku; Falta por ver en qué proporción se concreta esa renovación, pues la configuración de la ejecutiva es un sudoku difícil de cuadrar. El CORPES XXI señala que su repercusión es circunstancial o no significativa $(0,20$ casos por millón para la forma $s u$ doku) y su distribución es panhispánica:

\begin{tabular}{|lrr|}
\hline País & Freq & Fnorm. \\
\hline \hline España & 16 & 0,18 \\
\hline Argentina & 9 & 0,41 \\
\hline Ecuador & 7 & 1,20 \\
\hline Chile & 4 & 0,28 \\
\hline Colombia & 4 & 0,23 \\
\hline México & 4 & 0,14 \\
\hline Puerto Rico & 3 & 0,75 \\
\hline Venezuela & 3 & 0,25 \\
\hline República Dominicana & 1 & 0,17 \\
\hline
\end{tabular}
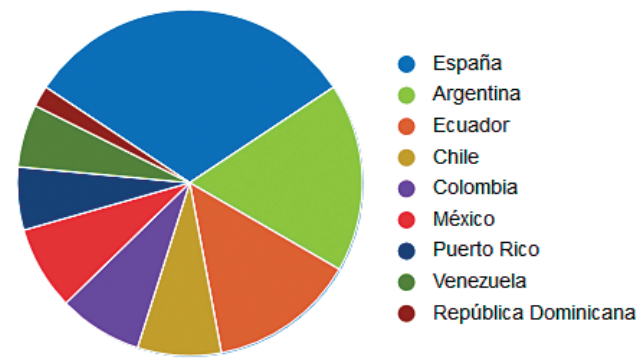

GRÁFICA 5 .

Distribución por países. Fuente: CORPES XXI.

\subsection{Desastres naturales}

\subsection{1. «sunami»}

El japonesismo «sunami» procede de la voz 津波, [tsunam'i], que en japonés se utiliza para referirse a las 'olas de enorme amplitud y frecuencia creadas a raíz de un terremoto de la erupción de un volcán submarino. Al acercarse a la costa, aumentan rápidamente de altura, haciéndose anormalmente elevadas en puertos y en el interior de las bahías' (DaiD). 
La transcripción preferida por los corpus españoles (CORDE, CREA y CORPES XXI) es la extranjerizante, tsunami $(s)^{37}$. Esta misma tendencia observamos en las obras lexicográficas españolas (DEA, DUEAE, NDVUA, DUE, DClave y DLE 2014) ${ }^{38}$ y extranjeras (MWCD, OED, DFL, LPR, DHLP, DPLP, DOVLI y Zingarelli). El elemento extranjerizante $t s u$ - ha dado en español dos posibles articulaciones: (1) una fiel a la grafía, esto es, se articula una dentoalveolar, africada, [ts]; (2) se elimina la oclusión y se simplifica a nuestra /s/ (DEA). Por la ausencia de marcas acentuales gráficas en los corpus y por los descriptores fónicos en $D E A$, sabemos que esta voz ha pasado al español con patrón paroxítono, similar al de la lengua japonesa.

Por lo que concierne al significado del término, detectamos acuerdo entre los corpus del español (CORDE, CREA y CORPES XXI) y las obras lexicográficas, tanto españolas (DEA, DUEAE, NDVUA, DUE, DClave y DLE 2014) como extranjeras (MWCD, OED, $D F L, L P R, D H L P, D P L P, D O V L I$ y Zingarelli). Todos han adoptado el término con el valor semántico de la voz japonesa original, es decir, lo emplean para referirse a las 'olas de enorme amplitud y frecuencia creadas a raíz de un terremoto de la erupción de un volcán submarino. Al acercarse a la costa, aumentan rápidamente de altura, haciéndose anormalmente elevadas en puertos y en el interior de las bahías' (DaiD). De este modo, como elemento natural catastrófico, suele estar asociado en los corpus a vocablos como: violentos, arrasar, enormes distancias, velocidad de la marea, mar, epicentro, terremotos, costas, movimientos sísmicos submarinos, inundaciones, seísmo, víctimas, etc. Por otro lado, puede emplearse también con sentido figurado ${ }^{39}$ : el PSOE ha resistido el pulso, salvo seísmo hoy de escala japonesa en las urnas, con tsunami posterior en el partido.

Según las obras lexicográficas españolas (DEA, DUEAE, NDVUA, DUE, DClave y $D L E$ 2014), esta voz ha sido adoptada con género masculino en español. En los corpus comprobamos que aparece junto a artículos masculinos (el-los, un), la amalgama de preposición y artículo $(\mathrm{al}, \mathrm{del})$ y otros adyacentes nominales (otro, asociado, asiático, famoso, catastrófico, registrados, devastadores, etc.). Con este género ha pasado a otras lenguas romances como el francés (DFL y $L P R)$, el portugués ( $D H L P$ y $D P L P)$ y el italiano (-invariable-: DOVLI y Zingarelli). En cuanto a la forma plural, aunque hallamos un gran número de muestras con morfema de plural /-s/ acompañado de artículo (los tsunamis), detectamos muestras de morfema cero: también en el Atlántico se han dado tsunami, reflejo, tal vez, de cierta inestabilidad morfológica. Por otro lado, observamos preferencia por los siguientes patrones combinatorios: los devastadores efectos del tsunami, las víctimas del tsunami, tsunami asiático / en Asia, las consecuencias del tsunami, alerta de tsunami. Asimismo, suele combinarse en oraciones cuyo núcleo puede ser alguno de los siguientes verbos: barrer, asolar, destruir, afectar, abatirse sobre, devastar, y otros similares.

De acuerdo con los datos del CORDE, el primer registro escrito de esta palabra en español data de 1927, en la obra española de Francisco Hernández-Pacheco, Geología fisio-

\footnotetext{
37 Únicamente detectamos en CORPES XXI 2 casos de «sunami».

38 En el DLE 2014 aparece «sunami», que remite a la forma extranjerizante en cursiva, tsunami.

39 En francés $(L P R)$ portugués $(D H L P)$ e italiano (Zingarelli) también se registran usos figurados de la voz.
} 
gráfica, con el significante plural tsunamis (los frecuentes y violentos tsunamis que la arrasaron en diversas ocasiones). No sería registrada en una obra lexicográfica española hasta 1999 , en el $D E A$. Desde su primer registro escrito no hemos detectado cambios formales, más allá de la pérdida de $t$ inicial de $t s u$ en dos muestras, ni semánticos.

En otras lenguas europeas, su datación es ligeramente anterior: así, en inglés, los primeros testimonios son de 1897, tanto en $M W C D$ como en $O E D$. La primera lengua románica en tomarla fue el francés, en 1915, de acuerdo con el $L P R$. Para el italiano, DOVLI y Zingarelli datan su primer registro en 1961. El DHLP ofrece el impreciso dato de siglo XX como datación de la palabra. Es probable, dada la grafía extranjerizante, que el sistema de romanización Hepburn actuara en el proceso de adopción de esta voz en lengua española.

Teniendo en cuenta la información de los párrafos precedentes, opinamos que la voz «sunami» pertenece al grupo de los japonesismos que se encuentran a medio camino entre la asimilación parcial y la asimilación total. Su grafía es extranjerizante y su valor semántico necesita ser explicado en numerosas muestras con marcas autonímicas: ola gigantesca, ola gigante; gran ola; maremoto; enormes olas llamadas por los japoneses tsunamis; olas denominadas tsunamis; nombre japonés introducido en la ciencia; provocó una ola sísmica o maremoto denominada "tsunami"; además de los entrecomillados o cursivas que denotan su estatus exógeno en el sistema ${ }^{40}$. Si bien, debido a los dos grandes «sunamis» acaecidos en los años 2004 (en el sur y sureste asiático) y en 2011 (en Japón), hemos detectado un gran número de casos relativos en las muestras del CORPES XXI, como se puede comprobar en la siguiente tabla que indica el período de distribución:

TABLA I.

Distribución de tsunami. Fuente: CORPES XXI.

\begin{tabular}{|lrr|}
\hline Período & Freq & Fnorm. \\
\hline \hline $2011-2015$ & 665 & 15,90 \\
\hline $2006-2010$ & 611 & 5,31 \\
\hline $2001-2005$ & 348 & 3,84 \\
\hline
\end{tabular}

De este modo, queda demostrado que la voz ha sido ampliamente difundida, por lo que se espera un mayor conocimiento semántico de esta. Esta vasta difusión perjudicó el término hispánico maremoto, que vio mermada su frecuencia de uso desde 2006:

\footnotetext{
40 De otro lado, observamos una morfología estable, sobre todo en singular, además de patrones sintácticocombinatorios consolidados.
} 
TABLA 2.

Distribución de maremoto. Fuente: CORPES XXI.

\begin{tabular}{|lrr|}
\hline Período & Freq & Fnorm. \\
\hline \hline $2001-2005$ & 295 & 3,25 \\
\hline $2006-2010$ & 182 & 1,58 \\
\hline $2011-2015$ & 99 & 2,36 \\
\hline
\end{tabular}

El CORPES XXI informa sobre su repercusión en el sistema lingüístico del español, que es de frecuencia moderada (forma tsunami: 6,56 casos por millón, frente a los 2,32 casos por millón para maremoto).

\begin{tabular}{|lrr|}
\hline País & Freq & Fnorm. \\
\hline \hline España & 532 & 6,27 \\
\hline Chile & 234 & 16,38 \\
\hline México & 141 & 5,21 \\
\hline Argentina & 101 & 4,65 \\
\hline Colombia & 86 & 5,01 \\
\hline Ecuador & 75 & 12,94 \\
\hline Venezuela & 63 & 5,31 \\
\hline Bolivia & 62 & 12,94 \\
\hline Uruguay & 54 & 9,75 \\
\hline Perú & 51 & 6,86 \\
\hline
\end{tabular}

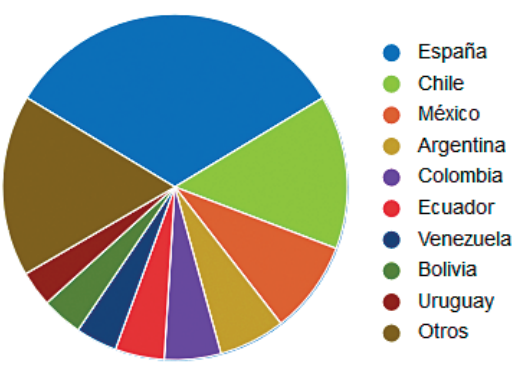

GRÁFICA 6.

Distribución por países para tsunami. Fuente: CORPES XXI.

\begin{tabular}{|lrr|}
\hline País & Freq & Fnorm. \\
\hline \hline España & 152 & 1,79 \\
\hline Chile & 136 & 9,52 \\
\hline México & 43 & 1,58 \\
\hline Colombia & 31 & 1,80 \\
\hline Venezuela & 28 & 2,36 \\
\hline Uruguay & 24 & 4,33 \\
\hline Argentina & 21 & 0,96 \\
\hline Ecuador & 21 & 3,62 \\
\hline Bolivia & 18 & 3,75 \\
\hline República Dominicana & 16 & 2,79 \\
\hline
\end{tabular}

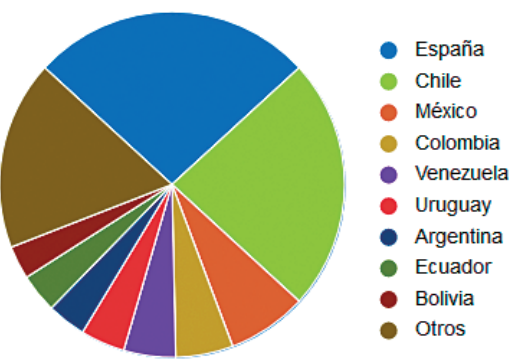

GRÁFICA 7.

Distribución por países para maremoto. Fuente: CORPES XXI. 


\section{CONCLUSIONES}

Debido a que resulta imposible ofrecer datos conclusivos para el cajón de sastre anterior, pues no constituye un área referencial unitaria, dividiremos sus componentes en subgrupos que sí conforman un campo nocional. De este modo, podemos extraer las siguientes conclusiones:

(a) Las voces relativas al área referencial de la geografía y los gentilicios («nipón»y «toquiota») son vocablos totalmente asimilados en el sistema léxico de la lengua española, con distribución panhispánica. Ambos pueden funcionar como sustantivo y como adjetivo, aunque, mientras que «nipón» presenta variación de género, «toquiota» funciona como sustantivo y adjetivo común. Los primeros registros de «nipón» proceden de la segunda mitad del siglo XIX y los de «toquiota», de la segunda mitad del XX. «Nipón» no ha sufrido más adaptación que la morfológicamente necesaria, pero «toquiota» sí ha pasado por un complejo proceso de sufijación - característica que sí comparte con otras lenguas romances, como el francés y el italiano-.

(b) Por lo que respecta a los japonesismos automovilísticos, «misubisi» y «toiota», han especializado su valor semántico y eso se ve reflejado en sus adaptaciones gráficas: ambos, con mayúscula inicial, hacen referencia a la compañía, mientras que con minúscula equivalen al vehículo de dicha marca — especialmente «toiota», ya que para «misubisi» también impera el uso de mayúscula con valor semántico de 'vehículo', mientras que en minúscula se utiliza para 'pastilla de droga'- La distribución geográfica de ambos vocablos es panhispánica; sin embargo, «misubisi» ha desarrollado un valor semántico metafórico en España, donde se emplea para aludir a un 'tipo de droga de diseño'. «Toiota» posee un mayor grado de adaptación que «misubisi» y, además, cuenta con una forma derivada, toyotismo, y una recurrencia elevada de combinaciones sintácticas. Los primeros registros de estas voces se ubican en la segunda mitad del siglo XX.

(c) El pasatiempo matemático, «sudocu», apenas con poco más de una década de vida en lengua española, se encuentra prácticamente asimilado, si no tenemos en cuenta la grafía extranjerizante, sudoku. Es esta transcripción, procedente del sistema anglosajón Hepburn, junto con la historia de su introducción en Occidente — gracias a los diarios británicos - la que nos hace pensar que la lengua inglesa pudo actuar como intermediaria entre el étimo japonés y la lengua española. No debemos olvidar, asimismo, que el patrón esdrújulo japonés ha sido readaptado en español con articulación paroxítona, tal vez porque el vocablo pasó de forma escrita y se comenzó a leer de acuerdo con la tendencia al patrón silábico llano de la lengua española. La distribución geográfica de este sustantivo masculino — con morfología estable- posee carácter panhispánico.

(d) Por último, el sustantivo «sunami», relacionado con los desastres naturales, ha experimentado un incremento de recurrencia en los últimos 15 años, especialmente después de los dos grandes «sunamis» que tuvieron lugar en 2004 (en el sur y sureste asiático) y en 2011 (en Japón). Esto provocó que la frecuencia de su análogo 
patrimonial, maremoto, disminuyera desde 2006. Pese a su reciente introducción, esta voz ya cuenta con usos metafóricos y con patrones combinatorios y morfológicos estables - a excepción de ciertos casos con morfema de plural cero- . Parece que la RAE no ha tratado de poner fin al doblete articulatorio y transcriptivo tsunami-sunami en la última edición del DLE: ya que la solución sunami remite a tsunami.

\section{MATERIALES BIBLIOGRÁFICOS}

\subsection{Libros, manuales y artículos}

Akamatsu, Tsutomu (1997): Japanese Phonetics: Theory and Practice. Múnich: LINCOM Europa. Alvar, Manuel et al. (1967): Enciclopedia lingüística hispánica. Tomo II. Madrid: CSIC.

Álvarez de Miranda, Pedro (2004): «El léxico español, desde el siglo XVIII hasta hoy». En: Rafael Cano (coord.). Historia de la lengua española. Barcelona: Ariel, 1037-1064.

Cid Lucas, Fernando (2009): «La presencia de palabras japonesas en el castellano: una lectura antropológica de su incorporación». Observatorio de la Economía y la Sociedad del Japón, 1(6).

DoI, SCHun (2013): Japanese Loanwords Found in the Oxford English Dictionary and Kaempfer's the History of Japan. Tesis doctoral. Japón: Universidad de Nagoya.

Fernández Mata, RAFAel (2015): Los japonesismos de la lengua española: Historia y transcripción. Tesis doctoral. Sevilla: Universidad Pablo de Olavide.

- (2017): «Los japonesismos del español actual». Revista de Filología de la Universidad de La Laguna, 35, 149-168.

- (20I8): «MÉtOdO DE TRANSCRIPCión DEL JAPONÉS AL ESPAÑol». Onomázein, 42, páginas por determinar.

Fradejas Rueda, José Manuel (2000): Fonología histórica del español. Madrid: Visor Libros.

Frago Gracia, José Antonio (1997): «Japonesismos entre Acapulco y Sevilla: sobre biombo, catana y maque». Boletín de Filología de la Universidad de Chile, 36, 101-118.

Giménez FolQués, DAvid (2012): «Los extranjerismos en el español académico del siglo XXI». Normas. Revista de Estudios Lingüísticos Hispánicos, Anejo n. ${ }^{\circ}$ 3, 1-79.

Gómez CAPUZ, JuAn (1998): El préstamo lingüístico: conceptos, problemas y métodos. Cuadernos de Filología. Valencia: Universitat de València.

- (2004): Préstamos del español: lengua y sociedad. Madrid: Arco/Libros.

- (2005): La inmigración léxica. Madrid: Arco/Libros.

Kim, Tai Wham (1992): «Análisis lingüístico de los japonesismos en Triunfo de la fee en los reynos de Japón, de Lope de Vega». En M. Ariza Viguera (Ed.), Actas del II Congreso Internacional de Historia de la Lengua Española. Tomo I (pp. 1355-1358). Sevilla: Pabellón de España.

Knowlton, Edgar Colby (1959): Words of Chinese, Japanese, and Korean Origin in the Romance languages. Tesis doctoral. Estados Unidos: Stanford University.

Labrune, Laurence (2012): The Phonology of Japanese. Oxford: Oxford University Press.

LAPESA, RAFAel (2005 [1981']): Historia de la lengua española. Madrid: Gredos.

Prieto Vera, Luis (2007): «Voces de origen japonés en el léxico de la prensa de Santiago de Chile». Boletín de filología, 42, 157-318.

Quilis, Antonio (2006 [1993]): Tratado de fonología y fonética españolas. Madrid: Gredos.

Real Academia Española (2009): Nueva gramática de la lengua española. Madrid: Espasa.

- (2010): Ortografía de la lengua española. Madrid: Espasa. 
- CORDE. Corpus diacrónico del español. [en línea]: <http://corpus.rae.es/cordenet.html> [Consulta: 20/12/2017].

- CREA. Corpus de referencia del español actual. [en línea]: <http://corpus.rae.es/creanet.html> [Consulta: 20/12/2017].

- CORPES XXI. Corpus del español del siglo XXI. [en línea]: <http://web.frl.es/CORPES/view/ inicioExterno.view> [Consulta: 20/12/2017].

- NTLLE. Nuevo Tesoro Lexicográfico de la Lengua Española. [en línea]: <http://ntlle.rae.es/ntlle/ SrvltGUILoginNtlle> [Consulta: 20/12/2017].

Reyes Díaz, María Josefa (2004): «A propósito de léxico y cultura». En S. Bravo Utrera (Ed.), Traducción, Lenguas, Literaturas. Sociedad del conocimiento. Enfoques desde y hacia la cultura (pp. 221-233). Las Palmas de Gran Canaria: Servicio de Publicaciones de la Universidad de Las Palmas de Gran Canaria.

Tsujimura, Natsuko (1996 [2007]): An Introduction to Japanese Linguistics. Malden MA: Blackwell Publishing.

Vance, Timothy J. (2008): The Sounds of Japanese. Cambridge: Cambridge University Press.

\subsection{Obras lexicográficas hispánicas y extranjeras}

Almarza, Nieves et al. (2012): Diccionario Clave: diccionario de uso del español actual. [en línea]: $<$ http://clave.smdiccionarios.com/app.php> [Consulta: 24/03/2018].

Alvar EzQuerra, Manuel (director) (1994): Diccionario de voces de uso actual. Madrid: Arco/ Libros.

- (director) (2003): Nuevo diccionario de voces de uso actual. Madrid: Arco/Libros.

Cannella, Mario y Beata Lazzarini (directores) (2014): Lo Zingarelli 2015: Vocabolario della lingua italiana. Bolonia: Zanichelli. [en línea]: <http://dizionari.zanichellipro.it/> [Consulta: 24/03/2018].

Corominas, Joan y Pascual, José Antonio (2012 [1991-1997]): Diccionario crítico etimológico castellano e hispánico. Madrid: Gredos [CD-ROM].

Devoto, Giacomo y Oli, Gian Carlo (2012): Il Devoto-Oli: vocabolario della lingua italiana 2013. Firenze: Le Monnier [CD-ROM].

Houaiss, AntôNio (director) (2001): Dicionário Houaiss da Língua Portuguesa. Río de Janeiro: Editora Objectiva.

Jeuge-Maynart, Isabelle (directora) (2016): Dictionnaire de français Larousse. París: Hachette Livre - Département Informatique Groupe Livre (DSI). [en línea]: <http://www.larousse.fr/dictionnaires/francais-monolingue> [Consulta: 26/03/2018].

Lahuerta Galán, Javier (director) (2003): Diccionario de uso del español de América y España. Barcelona: Vox [CD-ROM].

Matsumura, AkiRa (director) (2006): Daiyirín. Toquio: Sanseido Books. [en línea]: <www.kotobank. jp> [Consulta: 24/03/2018].

- (director) (2008): Daiyisén Digital. Toquio: Siogacucan. [en línea]: <www.kotobank.jp> [Consulta: 24/03/2018].

Moliner, María (2008 [1996]): Diccionario de uso del español. Madrid: Gredos [CD-ROM].

Priberam Informática, S.A., (ed.) (2008-2013): Dicionário Priberam da Língua Portuguesa. [en línea]: $<$ http://www.priberam.pt/dlpo/> [Consulta: 26/03/2018].

Real ACAdemia Española (2001): Diccionario de la Real Academia Española. Madrid: Espasa Calpe [CD-ROM].

- (2005): Diccionario panhispánico de dudas. Madrid: Santillana. [en línea]: <http://lema.rae.es/ $\mathrm{dpd} />$ [Consulta: 26/03/2018]. 
- (2014): Diccionario de la Real Academia Española. Madrid: Espasa Libros. [en línea]: <http:// www.rae.es/> [Consulta: 26/03/2018].

Rey, AlAin (director) (2014) : Le Petit Robert de la langue française. Francia: Le Robert [CD-ROM].

SÁnChez, Aquilino (2006 [2001]): Gran diccionario de uso del español actual. Madrid: SGEL [CD$\mathrm{ROM}]$.

Seco, Manuel, Andrés, Olimpia y Ramos, Gabino (1999): Diccionario del español actual. Madrid: Aguilar.

Simpson, John (director) (2009): Oxford English Dictionary. Oxford University Press [CD-ROM, segunda edición 4.0.].

The Merriam-Webster (2003): Merriam-Webster's Collegiate Dictionary. Springfield: Merriam-Webster [undécima edición] [CD-ROM].

\section{ANEXO: LISTADO DE ABREVIACIONES EMPLEADAS}

$\begin{array}{ll}\text { CORDE } & \text { Corpus diacrónico del español } \\ \text { CORPES XXI } & \text { Corpus del español del siglo XXI } \\ \text { CREA } & \text { Corpus de referencia del español actual } \\ \text { DaiD } & \text { デジタル大辞泉 > Daiyisén Digital } \\ \text { Daiyirín } & \text { 大辞林 > Daiyirín } \\ \text { DClave } & \text { Diccionario Clave: diccionario de uso del español actual } \\ \text { DCECH } & \text { Diccionario crítico etimológico castellano e hispánico } \\ \text { DEA } & \text { Diccionario del español actual } \\ \text { DFL } & \text { Dictionnaire de Français Larousse } \\ \text { DHLP } & \text { Dicionário Houaiss da Língua Portuguesa } \\ \text { DLE } & \text { Diccionario de la lengua española } \\ \text { DPLP } & \text { Dicionário Priberam da Língua Portuguesa } \\ \text { DOVLI } & \text { Il Devoto-Oli: vocabolario della lingua italiana } \\ \text { DUE } & \text { Diccionario de uso del español } \\ \text { DUEAE } & \text { Diccionario de uso del español de América y España } \\ \text { DVUA } & \text { Diccionario de voces de uso actual } \\ \text { GDUEA } & \text { Gran diccionario de uso del español actual } \\ \text { LPR } & \text { Le Petit Robert de la langue française } \\ M W C D & \text { Merriam-Webster's Collegiate Dictionary } \\ \text { NDVUA } & \text { Nuevo diccionario de voces de uso actual } \\ \text { NTLLE } & \text { Nuevo Tesoro Lexicográfico de la Lengua Española } \\ \text { OED } & \text { Oxford English Dictionary } \\ \text { Zingarelli } & \text { lo Zingarelli 2015. Vocabolario della lingua italiana } \\ \end{array}$


